Int. J. Dev. Biol. 58: 45-50 (2014)

doi: $10.1387 / \mathrm{ijdb} .130261 \mathrm{jb}$

\title{
Expression pattern of zcchc24 during early Xenopus development
}

\author{
MARTA VITORINO ${ }^{1,2}$, ELIZABETH CORREIA ${ }^{1,2}$, ANA-RITA SERRALHEIRO ${ }^{2}$, ANA-CATARINA DE-JESUS ${ }^{1,2}$, \\ JOSÉ M. INÁCIO ${ }^{1,2}$ and JOSÉ A. BELO*,1,2,3
}

\begin{abstract}
${ }^{1}$ Regenerative Medicine Program, Departamento de Ciências Biomédicas e Medicina, Universidade do Algarve, ${ }^{2}$ IBBInstitute for Biotechnology and Bioengineering, Centro de Biomedicina Molecular e Estrutural, Universidade do Algarve, Campus de Gambelas, Faro and ${ }^{3}$ Faculdade de Ciências Médicas, Universidade Nova de Lisboa, Lisboa, Portugal.
\end{abstract}

\begin{abstract}
We report the expression pattern of a novel Xenopus laevis gene, zcchc24, which encodes a protein containing two zinc finger domains from the zf-CCHC and zf-3CxxC superfamilies. This protein shares $>84 \%$ amino acid identity with its vertebrate homologues. During $X$. laevis embryonic development, zcchc24 is expressed at gastrula stages in the dorsal mesoderm, including the cardiac precursors region. During neurula stages, zcchc24 is expressed as two stripes in the dorsal region, more precisely, in the somitogenic mesoderm until the cardiac mesoderm. At early tailbud stages, zcchc24 continues to be expressed in these regions, but starts to be expressed in the migrating neural crest. Later, this gene is expressed in the head, branchial arches, heart and somites. The zinc finger domains present in Zcchc24 protein and its dynamic gene expression pattern suggest that Zcchc24 might be involved in the regulation of heart, somites and of branchial arch formation/patterning, namely in the regulation of apoptosis.
\end{abstract}

KEY WORDS: zcchc24, zinc finger, heart development, somitogenic mesoderm, neural crest

The circulatory system is the first one to become functional during vertebrate embryo development, and is composed by the heart, blood cells and vessels. The formation of the heart is a wellconserved process among vertebrate, however, the molecular mechanisms involved in it are not well defined. To address this limitation, a differential screening for genes expressed in the heart precursor cell lineages of chick embryos was performed in our lab (Bento et al., 2011). From the 777 detected genes, 199 were classified as upregulated uncharacterized genes. Among them, it was obtained ZCCHC24(zinc fingerdomain-containing protein 24), which predicted amino acid sequence is identical $90.8 \%, 90.5 \%$ and $90.8 \%$ to its human, mouse and frog homologs, respectively (Bento et al., 2011).

Bioinformatic analysis showed that Xenopus laevis zcchc24 (Genbank accession no. KF438010) encodes a 239 amino acids protein, with a predicted molecular mass of $26.99 \mathrm{kDa}$, and with two zinc finger domains (Fig. 1, http://www.ncbi.nlm.nih.gov/Structure/ cdd/wrpsb.cgi). Zinc fingers are relatively small protein domains that bind to zinc atoms and, normally, contain finger-like protrusions that make contact with their target molecules. They were initially identified as DNA-binding motifs but several studies showed that these domains can bind to DNA, RNA, proteins and lipids (Hall, 2005). Indeed, proteins with zinc finger domains are extremely abundant in eukaryotic genomes but can vary both in structure, as well as in function. More, they are involved in biological functions as diverse as cell growth, differentiation, DNA recognition, RNA packaging, transcriptional activation, regulation of apoptosis, protein folding and assembly, and lipid binding (Laity et al., 2001).

Consequently, there are several superfamilies of zinc finger motifs, which are classified according to its sequence and structure (Krishna et al., 2003). Zcchc24 protein contains two different zinc finger domains: one associated to the zf-CCHC superfamily and the other to the zf-3CxxC superfamily (Fig. 1). A typical example of proteins containing the $\mathrm{zf}-\mathrm{CCHC}$ domain is the inhibitor of apoptosis (IAP) that has been reported as a regulator of programmed cell death by inhibition of caspases (Krishna et al., 2003). The zf$3 \mathrm{CxxC}$ domain is present in several proteins with functions related with modifications in either DNA or chromatin, such as histone $\mathrm{H} 3$ lysine 36, and demethylases KDM2A and B (Birke et al., 2002, Blackledge et al., 2010).

Abbreviations used in this paper: ZCCHC24, zinc finger domain-containing protein 24.

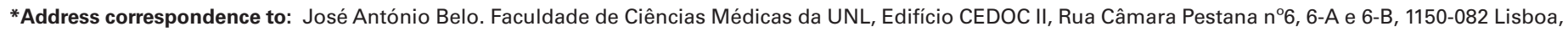
Portugal.Tel: +351-218-803-102. Fax+351-218-803-010. E-mail: jose.belo@fcm.unl.pt
}

Accepted: 19 December 2013. Final, author-corrected PDF published online: 30 April 2014.

ISSN: Online 1696-3547, Print 0214-6282 
To analyze the potential function of $X$. laevis zcchc24 during early frog embryo development, we examined its expression by whole-mount in situ hybridization (WISH).

zcchc24 transcripts were first detected at midblastula stage, in both dorsal and ventral marginal zones of the embryos (Fig. 2 $\left.A, A^{\prime}\right)$, showing a ring around the marginal zone or presumptive mesoderm. Then, at the onset of gastrulation, zcchc24 expression was observed in the dorsal mesoderm of both involuting marginal zone (IMZ), a region immediately above the dorsal blastopore lip, and non-involuting marginal zone (NIMZ), which is the region on the top of IMZ (Fig. 2 B,B').

During gastrulation, and more specifically at stage (st) 11-12, zcchc24 is expressed in the dorsal mesoderm, in the somitogenic mesoderm, excluding the dorsal midline (Fig. 2 C C',E,E'). myoD, whose expression has been described in the same region, was used as a marker of somitogenic mesoderm (Fig. $2 \mathrm{D}, \mathrm{F})$. This gene is involved in the formation of somites, and the knock-down of the MyoD disrupts the correct alignment of muscle fibers (Maguire et al., 2012). At this stage, zcchc24 is also expressed in two lateral mesoderm stripes around the blastopore that culminate in the dorsal side of the embryo (Fig. 2 C,E). Interestingly, these two lateral mesodermal stripes are correlated with the region in which the heart is originated. As a matter of fact, it has been suggested that, during gastrulation, the precardiac mesoderm migrates in two bilateral heart field located in the anterior lateral mesoderm (Sater and Jacobson, 1989).

From mid (st 11) to the end of gastrulation/ beginning of neurulation (st 13), zcchc24 expression seems to follow the migration of involuting mesoderm along the anteroposterior axis (Fig. 3A). During neurulation, zcchc24 expression is detected in the anterior and somitogenic mesoderm (Fig. 3 B-B",E), which later will give rise to lateral and neural plate, and the somites, respectively. At these stages, the expression of zcchc24 decreases progressively from the posterior to the anterior part of the embryo (Fig. 3 B,E). When compared with the early cardiac lineage marker

Fig. 1. Sequence alignment of Zcchc24 family members. Comparison of the predicted amino acid sequence of X. laevis Zcchc24 with its chick, human, mouse and rat homologs. X. laevis Zcchc24 (Genbankaccession no. KF438010) shares $90.8 \%$ of identity with chick ZCCHC24 (XP_421599), 86.3\% with human ZCCHC24 (NP_699198), 84.6\% with mouse ZCCHC24 (NP_001094903), and 84.2\% with ZCCHC24 rat (NP_001101864) homologs. Conserved residues are shaded in orange while identical amino acids among some of the sequences are shaded in light blue. The absence of residues at the corresponding region is indicated by dashes. Both zf-CCHCand zf-3CxxC zinc finger domains are displayed in green.

X.laevis

$n k x 2.5$, it is possible to observe that anteriorly zcchc24 expression is adjacent to the cardiac mesoderm, while more posteriorly, $z c$ chc24 is expressed in the paraxial mesoderm. (Fig. 3 C,D)

Afterwards, at early tailbud stages, zcchc24 expression is detected in the unsegmented somitogenic mesoderm and in the somites (Fig. 4A,B). In contrast, $n k x 2.5$ is predominantly expressed in the differentiating cardiac muscle (Fig. 4C). In addition, when we compare the expression of zcchc24 with the expression of twi, a neural crest marker, we observe that zcchc24 transcripts are in the migrating neural crest cells (Fig. 4 B,D,E).

At later tailbud stages, zcchc24 is expressed in the differentiating cardiac muscle, in the presumptive heart region and in the head, excluding the cleft between the branchial arches and cement gland (Fig. 4 F,G). A transverse section of a st 32 embryo showed

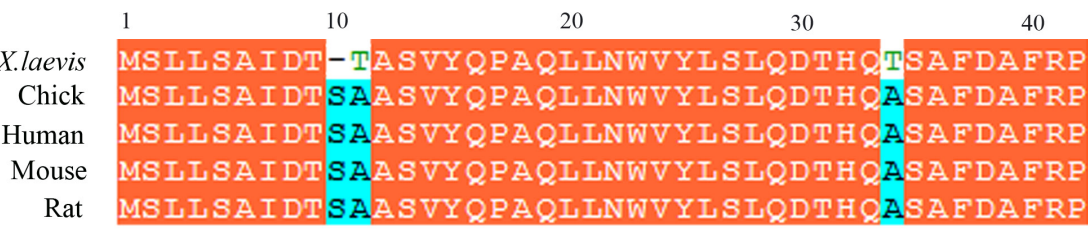
50

X.laevis

Chick

Human

Mouse

Rat

Chick

Human

Mouse

Rat

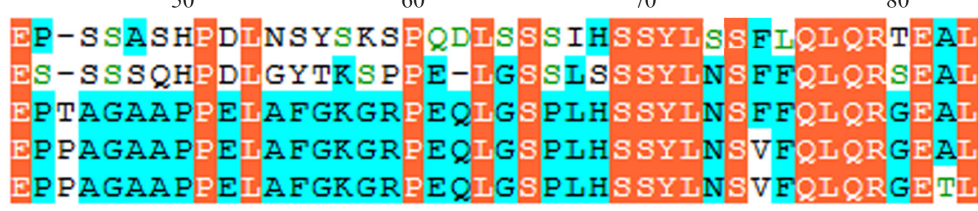

90

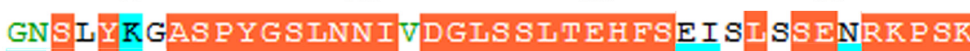
SSSIYKSASPY GSINNIVDGISSIIEH ESDLSISSEARKRSR SNSVYKGASPYGSINNIADGISSITEH ESDLTITSEARKPSR SS SVYRNASPYGSINNIADGISSITEH ESDLTITSEARKPSR SS SVYRNASPYGSINNIADGLSSITEHESDLTITSEARKPSK

zf-CCHC
140

X.laevis

Chick

Human

Mouse

Rat

X.laevis
Chick
Human
Mouse
Rat

RP P PNY I CHI C ENKGHY K DCPQARPK GE GITPYQGK KRCFG RP P PNYICHICENKGHYIKDCPQARPKGEGIT PYQGKRRCEG RP P PNY I CHIC ENKGHY I DCPQARPK GEGITPYQGKKRC EG RP P PNYLCHLCENKGHYIKDCPQARPKGEGLT PYQGKRRCEG RPP PNY I CHICENKGHYIKDCPQARPKGEGITPYQGKKRCEG

zf-3CxxC

$\begin{array}{llll}170 & 180 & 190 & 200\end{array}$

EYKCPKCKRKWMSGNSWANMGQECIKCHINVYRHKQREIEKE

EYKCPKCKRKWMSGNSWANMGQECIKCHINVYPHKQRPI EKE EYKCPRCKRKWMSGNSWANMGQECIKCHINVYPHKQRPLEKE EYKCPKCKRKWMSGNSWANMGQECIKCHINVY RHKQRPIEKE EYKCPKCKRKWMSGNSWANMGQECIKCHINVYPHKQRPIEKP 41 42 42 42 42

82 82 84 84 84 $\begin{array}{rlll}\text { X.laevis } & \text { DGIDVSDQSK EHPQHICEKCKVIGYYCRRVQ } & 239 \\ \text { Chick } & \text { DGIDVSDQSKEHPQHICEKCKVIGYYCRRVQ } & 239 \\ \text { Human } & \text { DGIDVSDQSKEHPQHICEKCKVIGYYCRRVQ } & 241 \\ \text { Mouse } & \text { DGIDVSDQSKEHPQHICEKCKVIGYYCRRVQ } & 241 \\ \text { Rat } & \text { DGLDVSDQSKEHPQHICEKCKVIGYYCRRVQ } & 241\end{array}$

zf-3CxxC

\section{$\mathrm{zf}-3 \mathrm{CxxC}$}



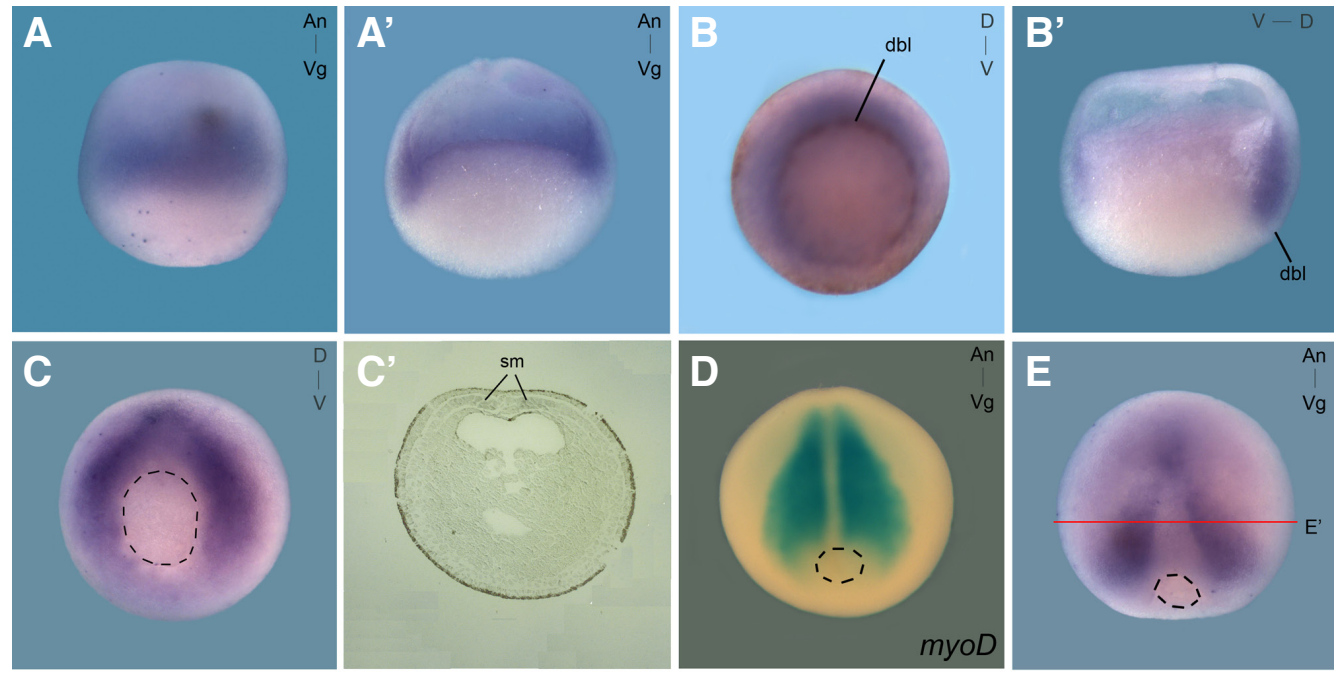

Fig. 2. zcchc24 expression from blastula to gastrula stages. Whole mount in situ hybridization using DIG labelled zcchc24 (A-C', E,E') or fluorescein labelled myoD (D,F) was performed on embryos from blastula to gastrula stages. $\left(\mathbf{A}, \mathbf{A}^{\prime}\right)$ At blastula stages, zcchc24 is expressed as a ring around the marginal zone in both dorsal and ventral sides. The embryos
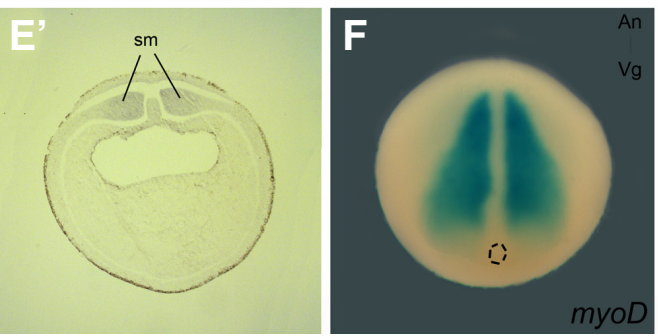

display the animal hemisphere to the top. (B, $\left.\mathbf{B}^{\prime}\right)$ In the beginning of gastrulation (st 10.5), zcchc24 expression is restricted to the dorsal mesoderm in both involuting and non-involuting marginal zone. (C,C', E,E') At late gastrula (st 11 and 12), zcchc24 transcripts are detected in two lateral mesoderm stripes around the blastopore that culminate in the dorsal mesoderm, the somitogenic mesoderm, but is excluded from the midline. (D,F) myoD is expressed in the somitogenic mesoderm. ( $\left.\mathbf{A}^{\prime}, \mathbf{B}^{\prime}\right)$ Hemisections of $(\mathbf{A}, B)$, respectively. (C', $\left.\mathbf{E}^{\prime}\right)$ Transverse sections of st 11 and st 12 embryos, respectively, with dorsal side displayed to the top. $(\mathbf{B}, \mathbf{C})$ Vegetal views. (D,E,F) Dorsal views. Dashed lines delimitate the blastopore. An, animal; Vg, vegetal; $D$, dorsal; V, ventral; dbl, dorsal blastopore lip; sm, somitogenic mesoderm.

that $z c c h c 24$ transcripts are clearly detected in heart region, more precisely, in endocardium, myocardium and pericardium, and in branchial arch mesenchyme (Fig. 4G'). The expression of zcchc24 (Fig. 4G) in the heart is similar to the expression of $n k x 2.5$ in this region (Fig. $4 \mathrm{H}, \mathrm{I}$ ). More, when zcchc24 and twi expressions are compared, it becomes obvious that both genes are expressed in the branchial arches (Fig. $4 \mathrm{~J}, \mathrm{~K}$ ).

According to our data, the expression pattern of Xenopus zcchc24 is in part similar to the expression pattern of its chick homolog.
Here, we show that, early in development, Xenopus zcchc24 is expressed in the dorsal mesoderm, in which the cardiac mesoderm is initially formed, and later is expressed in the head, heart and somites, like its chick homolog at Hamburger and Hamilton stage 10 (HH10; Fig. 5). This suggests that these homologs might have a role in heart formation, both in Xenopus and chick. Moreover, it demonstrates that zcchc24 expression pattern was conserved during evolution.

Several zinc finger proteins with a role in heart development
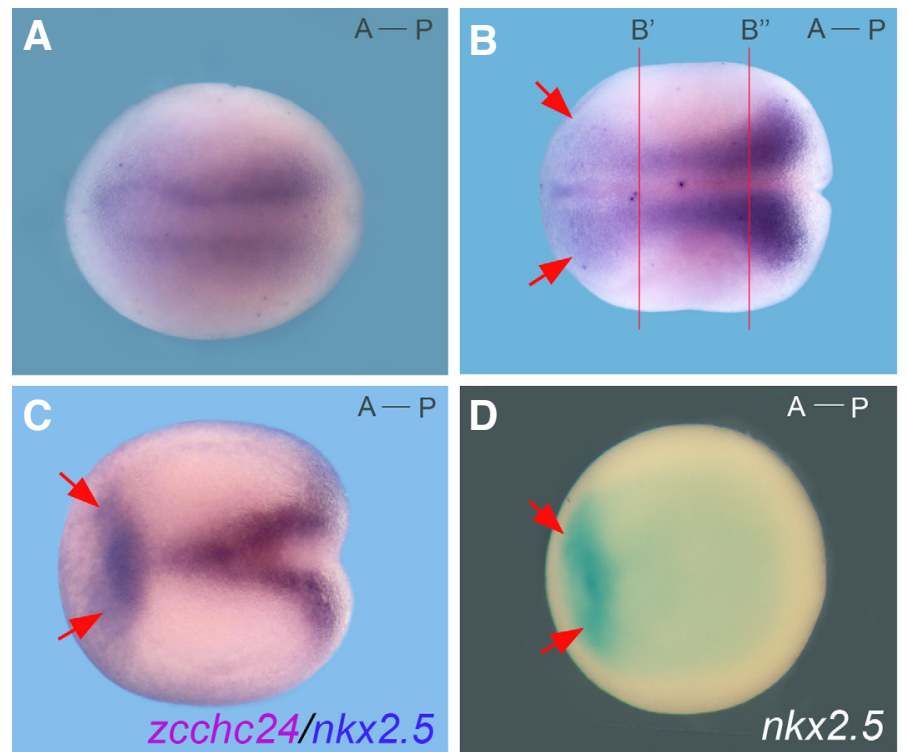
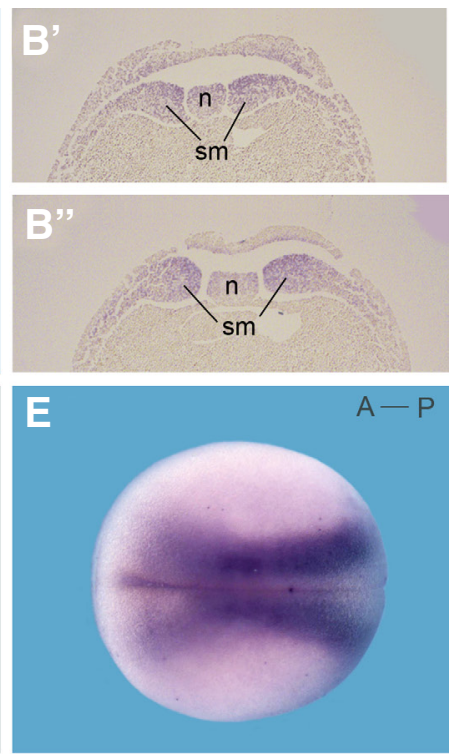

Fig. 3. zcchc24 expression during neurula stages. Whole mount in situ hybridization using DIG labelledzcchc24 (A-B", E) or fluorescein labelled nkx2.5 (D) and double whole mount in situ hybridization with DIG labelledzcchc24 and fluorescein labelled nkx2.5 (C) were performed on embryos at neurula stages. (A) At late gastrula/early neurula (st 13), zcchc24 is expressed as two stripes in the dorsal side of the embryo along the antero-posterior axis but is excluded from the notochord. (B-B", E) During neurula stages, zcchc24 is expressed in anterior and somitogenic mesoderm, decreasing the expression from the posterior to the anterior region. Comparison between the expression of zcchc24 and nkx2.5 (B,C,D) shows that the most anterior expression of

zcchc24 is adjacent to the cardiac mesoderm. (A,B, C-E) Embryos in a dorsal view. (B', B") Transversal sections of st 15 embryos with the dorsal region displayed to the top. Red arrows indicate the heart precursor region. A, anterior; P, posterior; $n$, notochord; sm, somitogenic mesoderm. 

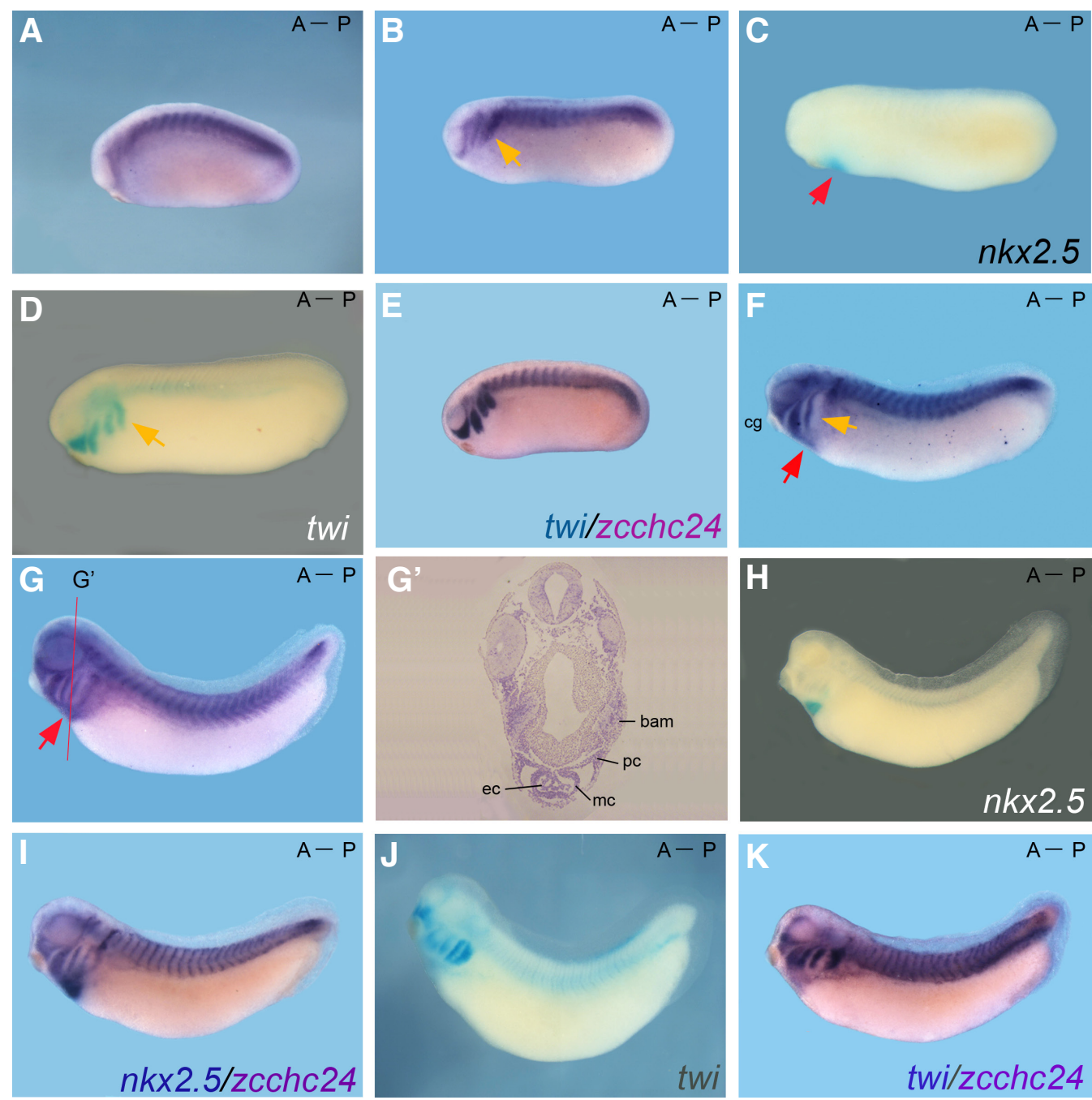

have been described. The GATA zinc finger-containing transcription factors are a family of proteins that have been implicated in regulation of gene expression in the heart development (Haworth et al., 2008). It was demonstrated in zebrafish that GATA5 is necessary for the production of the correct number of myocardial
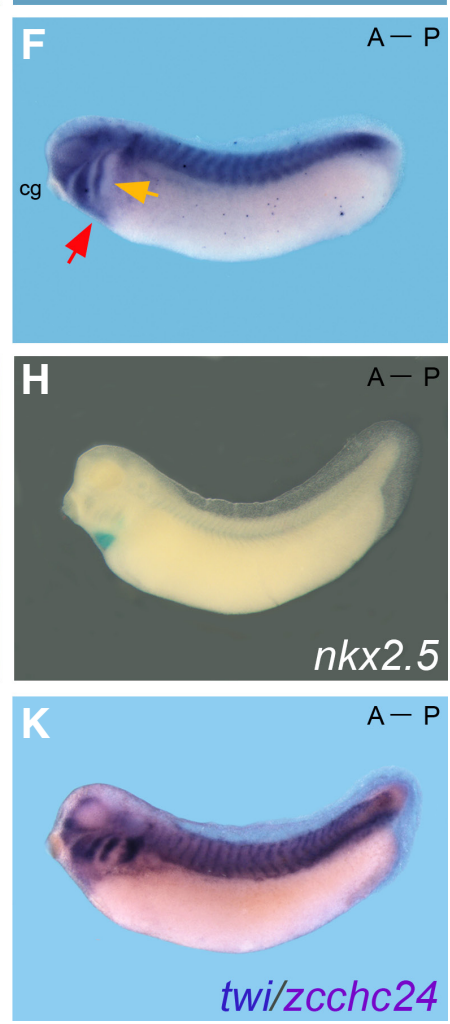

Fig. 4. Expression pattern of zcchc24 during tailbud stages. Whole mount in situ hybridization using DIG labelled zcchc24 (A, B, F-G'), fluorescein labelled $\mathrm{nkx2.5}(\mathbf{C}, \mathbf{H})$ or fluorescein labelled twi (D,J), and double whole mount in situ hybridization using DIG labelled zcchc24 and fluorescein labelled twi (E,K) or DIG labelled zcchc24 and fluorescein labelled $\mathrm{nk} \times 2.5$ (I) were performed on embryos at tailbud stages. (A-E) At early tailbud stages, zcchc24 is strongly expressed in the unsegmented somitogenic mesoderm, somites, and migrating neural crest but is absent in the region of differentiating cardiac muscle. (F) Later, at st 28, zcchc24 transcripts start to be detected in the forming heart, and in the entire head except the region between the branchial arches. (G-K) This expression is maintained throughout tailbud development, beingzcchc24 expressed in the somites, branchial arches, head and heart. All the embryos are displayed in a lateral view with the anterior region to the left. (G') Transversal section of the heart region of a st 32 embryo. The orange arrows indicate the neural crest/ branchial arches, and the red arrows indicate heart-forming region/heart. A, anterior; P, posterior; ec, endocardium; pc, pericardium; mc, myocardium; bam, branchial arch mesenchyme; cg, cement gland.

precursors and for the correct expression of several cardiac genes including $n k x 2.5$. Indeed, the overexpression of GATA5 induces contractile heart-like tissue (Reiter et al., 1999). GATA4 is another GATA family zinc finger that is also implicated in heart and liver development. It was shown that the knock-down of this transcript affects heart and liver primordia following their specification (Haworth et al., 2008, Holtzinger and Evans, 2005). In addition, after heart specification, GATA4 interacts with another GATA family member, GATA6, during its action in the development of heart in mouse, Xenopusand zebrafish (Holtzinger and Evans, 2005, Peterkin et al., 2003, Zhao et al., 2005). Therefore, since zcchc24 is expressed in heart precursor cells and later in the heart (Fig. 6), like GATA family genes, it is tempt fate to extrapolate a role for zcchc24 in the formation of the heart.

Nevertheless, zcchc24 is also highly expressed in somitogenic mesoderm and later in the somites. Sev-

Fig. 5. In chicken embryos, $\mathrm{ZCCHC24}$ is expressed in the heart and somites. Whole mount in situ hybridization using DIG labelled ZCCHC24 in chick embryos at HH10. (A, A') At this stage, ZCCHC24 is expressed in the heart (red arrow), somites (yellow arrow) and in head mesenchyme. (A') Magnification of (A). 


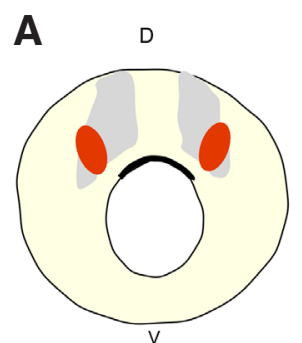

st 11

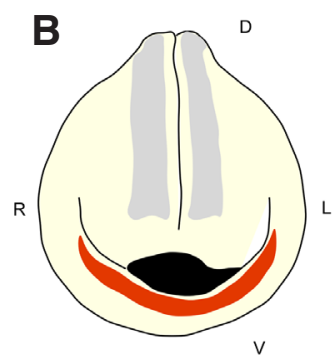

st 19

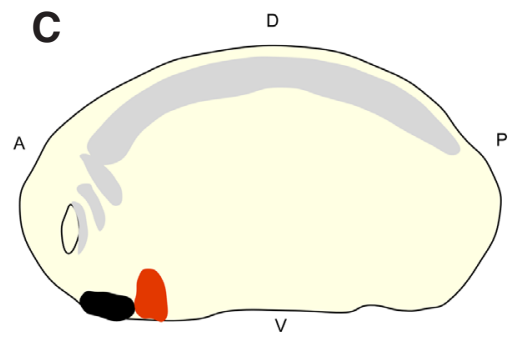

st 23
D

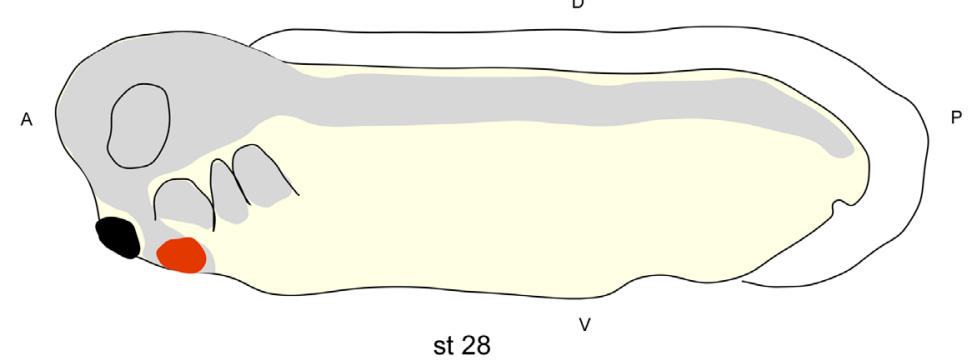

Fig. 6. Schematic representation of the localization of heart precursors and zcchc24 expression during $\boldsymbol{X}$. laevis embryonic development. Red areas represent the heart precursors and the gray areas represent the expression of zcchc24. (A) Vegetal view, (B) anterior view, (C,D) lateral views with the anterior region to the left. $A$, anterior; $P$, posterior; $D$, dorsal; $V$, ventral; $R$, right; L, left.

eral zinc fingers proteins like Gli, Gli3 and Gli4 members of the Hedgehog (Shh) signaling pathway have been implicated in somite formation. Gli-type proteins function as transcriptional repressors that respond to Shh signals, and control the expression of Shh-responsive genes such as myf5, the muscle master regulator (Hui and Angers, 2011). Therefore, we think that Zcchc24 putative role in the regulation of the proper somite segmentation could not be excluded.

The expression of zcchc24 in the migrating neural crest and later in the branchial arches suggests that zcchc24 might have a function during the development and/or migration of this tissue. Curiously, several zinc finger proteins of the Snail family have been associated to the neural crest formation (del Barrio and Nieto, 2002, Nieto et al., 1994). For example, it was reported that slug and snail, two members of this family, are important for neural crest specification and migration. In Xenopus embryos or animal caps, the overexpression of snail is able to induce the expression of slug among other neural crest markers such as foxD3, twi and ets1. On the other hand, slug is not able to induce these neural crest markers, however, gain-of-function studies performed in chick showed that slug overexpression increases cranial neural crest production, and its loss-of-function inhibits neural crest migration (Aybar et al., 2003, del Barrio and Nieto, 2002, Nieto et al., 1994).

Taken together, our results showed that zcchc24 is expressed mainly in three different precursors/structures: cardiac precursors/ heart, somitogenic mesoderm/somites, and neural crest/branchial arches. Curiously, several proteins of the zf-CCHC superfamily of zinc finger containing proteins were described to have a role on cell death inhibition. Moreover, the proper formation of the heart, branchial arches and somites requires none or a low level of apoptosis. High levels of apoptosis in these three structures have been reported to be responsible for defects (Graham et al., 1996, Kang and Izumo, 2000, Sanders and Parker., 2001). These observations indicate that Zcchc24 might be particularly important for the regulation of migration and/ or apoptosis. Nevertheless, further genetic and biochemical analysis must be performed to clarify the role of zcchc24 during $X$. laevis embryonic development.

\section{Materials and Methods}

\section{Xenopus embryo manipulations}

Xenopus eggs were obtained from females injected with $300 \mathrm{IU}$ of human chorionic gonadotropin (Sigma) and were fertilized in vitro. Eggs were dejellied with $2 \%$ cysteine hydrochloride, $\mathrm{pH} 8.0$. Embryos were grown in 0.1X MBS-H (1X MBS-H $=88 \mathrm{mM} \mathrm{NaCl}, 1 \mathrm{mM} \mathrm{KCl}, 2.4 \mathrm{mM} \mathrm{NaHCO} 3,0.82$ $\mathrm{mM}$ MgSO4, $0.41 \mathrm{mM} \mathrm{CaCl}$, $0.33 \mathrm{mM} \mathrm{Ca}(\mathrm{NO} 3) 2$, $10 \mathrm{mM}$ HEPES, $\mathrm{pH} 7.4,10 \mu \mathrm{g} / \mathrm{mL}$ streptomycin sulphate and $10 \mu \mathrm{g} / \mathrm{mL}$ penicillin) and staged according to Nieuwkoop and Faber (1967).

\section{Cloning of partial coding sequence of zcchc24 \\ Since the coding sequence (CDS) of zcchc24 was not available in stock centers, to obtain it, a}

partial coding sequence was isolated by RT-PCR. With this purpose, total RNA from stage 20 of Xenopus laevis embryos was isolated using trizol reagent according to the manufacturer's instruction. To perform the RT-PCR, first strand cDNA was synthesized using oligoDT hexamers as primers and zcchc24 CDS was amplified using a specific pair of primers (Forward 5'-CCATCCACTCCAGCTATCTGAGCA-3'; Reverse 5'-TTACTGAACACGGCGGCAGTAGTAGC-3'). The PCR product was cloned into pGEM ${ }^{\circledR}-T$ Easy and sequenced to confirm for correct DNA sequence.

\section{Whole mount in situ hybridization and histology}

Single and double whole mount in situ hybridization and anti-sense probes preparation was carried out as previously described (Belo et al., 1997). To generate the fluorescein labelled $n k x 2.5$, myoD and twi antisense RNA probes, plasmids containing fragments of these genes were linearized using Xbal, HindIII and EcoRI, respectively, and transcribed using T7 RNA polymerase. To synthesize zcchc24 DIG labelled probe, a plasmid containing zcchc24 fragment was linearized using Sall enzyme and transcribed using T7 RNA polymerase. Probes were purified using quick Spin Mini RNA columns (Roche). Hybridized RNAs were detected with alkaline phosphatase conjugated anti-DIG-antibody (Roche) and with alkaline phosphatase conjugated anti-Fluorescein-antibody (Roche) and developed with BM purple (Roche) or BCIP (Roche). Stained embryos were bleached by illumination in $1 \% \mathrm{H}_{2} \mathrm{O}_{2}, 4 \%$ formamide and $0.5 X$ SSC $\mathrm{pH}$ 7.0. Embryos were photographed under bright light using a MicroPublisher 5.0 RTV camera coupled with a Leica MZ16FA stereoscope or in a Zeiss Sterio Lumar V12 Stereomicroscope coupled with an Axiocam MRC.

For histology, after in situ hybridization, the Xenopus embryos were fixed overnight at $4^{\circ} \mathrm{C}$ in $4 \%$ PFA, embedded in paraffin, and sectioned in a Zeiss microtome. Sections were observed in a Zeiss Z2 microscope and photographed with a Zeiss AxioCam ICc3 camera.

\section{Acknowledgements}

$M$ Vitorino and J M Inácio are recipients of a F.C.T. Post-Doc fellowship with reference SFRH/BPD/65923/2009 and SFRH/BPD/41081/2007, 


\section{M. Vitorino et al.}

respectively. Elizabeth Correia is recipient of a F.C.TB.I. fellowship (PTDC/ SAU-BID/114902/2009). This work has been supported by research grants from F.C.T. and from IBB/CBME, LA to J.A. Belo in the frame of Project ref ${ }^{\circ}$ Pest-OE/EQB/LA0023/2013.

\section{References}

AYBAR, M.J., NIETO, M.A. and MAYOR, R. (2003). Snail precedes Slug in the genetic cascade required for the specification and migration of the Xenopus neural crest. Development 130: 483-494.

BELO, J., BOUWMEESTER, T., LEYNS, L., NKERTESZ, GALLO, M., FOLLETTIE, M. and ROBERTIS, E.D. (1997). Cerberus-like is a secreted factor with neutralizing activity expressed in the anterior primitive endoderm of the mouse gastrula. Mech Dev. Mech Dev 68: 45-57.

BENTO, M., CORREIA, E., TAVARES, A.T., BECKER, J.D. and BELO, J.A. (2011). Identification of differentially expressed genes in the heart precursor cells of the chick embryo. Gene Expression Patt. 11: 437-447.

BIRKE, M., SCHREINER, S., GARCÍA-CUÉLLAR, M.-P., MAHR, K., TITGEMEYER, F. and SLANY, R.K. (2002). The MT domain of the proto-oncoprotein MLL binds to CpG-containing DNA and discriminates against methylation. Nucleic Acids Res. 30: 958-965.

BLACKLEDGE, N.P., ZHOU, J.C., TOLSTORUKOV, M.Y., FARCAS, A.M., PARK, P.J. and KLOSE, R.J. (2010). CpG Islands Recruit a Histone H3 Lysine 36 Demethylase. Molec. Cell 38: 179-190.

DEL BARRIO, M.G. and NIETO, M.A. (2002). Overexpression of Snail family members highlights their ability to promote chick neural crest formation. Development 129: 1583-1593.

GRAHAM, A., KOENTGES, G. and LUMSDEN, A. (1996). Neural Crest Apoptosis and the Establishment of Craniofacial Pattern: An Honorable Death. Molec. Cell Neurosci. 8: 76-83.

HALL, T.M. (2005). Multiple modes of RNA recognition by zinc finger proteins. Curr.
Op. Struct. Biol. 15: 367-373.

HAWORTH, K., KOTECHA, S., MOHUN, T. and LATINKIC, B. (2008). GATA4 and GATA5 are essential for heart and liver development in Xenopus embryos. BMC Dev. Biol. 8: 74.

HOLTZINGER, A. and EVANS, T. (2005). Gata4 regulates the formation of multiple organs. Development 132: 4005-4014.

HUI, C.-C. and ANGERS, S. (2011). Gli Proteins in Development and Disease. Annu. Rev. Cell. Dev. Biol. 27: 513-537.

KANG, P.M. and IZUMO, S. (2000). Apoptosis and Heart Failure: A Critical Review of the Literature. Circ. Res. 86: 1107-1113.

KRISHNA, S.S., MAJUMDAR, I. and GRISHIN, N.V. (2003). Structural classification of zinc fingers: SURVEY AND SUMMARY. Nuc. Acid. Res. 31: 532-550.

LAITY, J.H., LEE, B.M. and WRIGHT, P.E. (2001). Zinc finger proteins: new insights into structural and functional diversity. Curr. Opin. Struct. Biol. 11: 39-46.

MAGUIRE, R.J., ISAACS, H.V. and ELIZABETH POWNALL, M. (2012). Early transcriptional targets of MyoD link myogenesis and somitogenesis. Dev. Biol. 371:256-268.

NIETO, M., SARGENT, M., WILKINSON, D. and COOKE, J. (1994). Control of cell behavior during vertebrate development by Slug, a zinc finger gene. Science 264: 835-839.

PETERKIN, T., GIBSON, A. and PATIENT, R. (2003). GATA-6 maintains BMP-4 and Nkx2 expression during cardiomyocyte precursor maturation. EMBO J 22.

REITER, J.F., ALEXANDER, J., RODAWAY, A., YELON, D., PATIENT, R., HOLDER, N. and STAINIER, D.Y.R. (1999). Gata5 is required for the development of the heart and endoderm in zebrafish. Genes Dev. 13: 2983-2995.

SANDERS, E. and PARKER., E. (2001). Ablation of axial structures activates apoptotic pathways in somite cells of the chick embryo. Anat Embryol (Berl) 204: 389-98.

SATER, A.K. and JACOBSON, A.G. (1989). The specification of heart mesoderm occurs during gastrulation in Xenopus laevis. Development 105: 821-830.

ZHAO, R., WATT, A.J., LI, J., LUEBKE-WHEELER, J., MORRISEY, E.E. and DUNCAN, S.A. (2005). GATA6 Is Essential for Embryonic Development of the Liver but Dispensable for Early Heart Formation. Molec. Cell Biol. 25: 2622-2631. 


\section{Further Related Reading, published previously in the Int. J. Dev. Biol.}

Sox17-dependent gene expression and early heart and gut development in Sox17-deficient mouse embryos

Sabine Pfister, Vanessa J. Jones, Melinda Power, Germaine L. Truisi, Poh-Lynn Khoo, Kirsten A. Steiner, Masami Kanai-Azuma, Yoshiakira Kanai, Patrick P. L. Tam and David A. F. Loebel

Int. J. Dev. Biol. (2011) 55: 45-58

http://dx.doi.org/10.1387/ijdb.103158sp

Building the vertebrate heart - an evolutionary approach to cardiac development José M. Pérez-Pomares, Juan M. González-Rosa and Ramón Muñoz-Chápuli

Int. J. Dev. Biol. (2009) 53: 1427-1443

http://dx.doi.org/10.1387/ijdb.072409jp

Myoskeletin, a factor related to Myocardin, is expressed in somites and required for hypaxial muscle formation in Xenopus

Hui Zhao, Martha L. Rebbert and Igor B. Dawid

Int. J. Dev. Biol. (2007) 51: 315-320

http://dx.doi.org/10.1387/ijdb.062260hz

Msx1 and Msx2 have shared essential functions in neural crest but may be dispensable in epidermis and axis formation in Xenopus

Deepak Khadka, Ting Luo and Thomas D. Sargent

Int. J. Dev. Biol. (2006) 50: 499-502

http://dx.doi.org/10.1387/ijdb.052115dk

Early stages of neural crest ontogeny: formation and regulation of cell delamination

Chaya Kalcheim and Tal Burstyn-Cohen

Int. J. Dev. Biol. (2005) 49: 105-116

http://dx.doi.org/10.1387/ijdb.041949ck

5 yr ISI Impact Factor $(2011)=2.959$
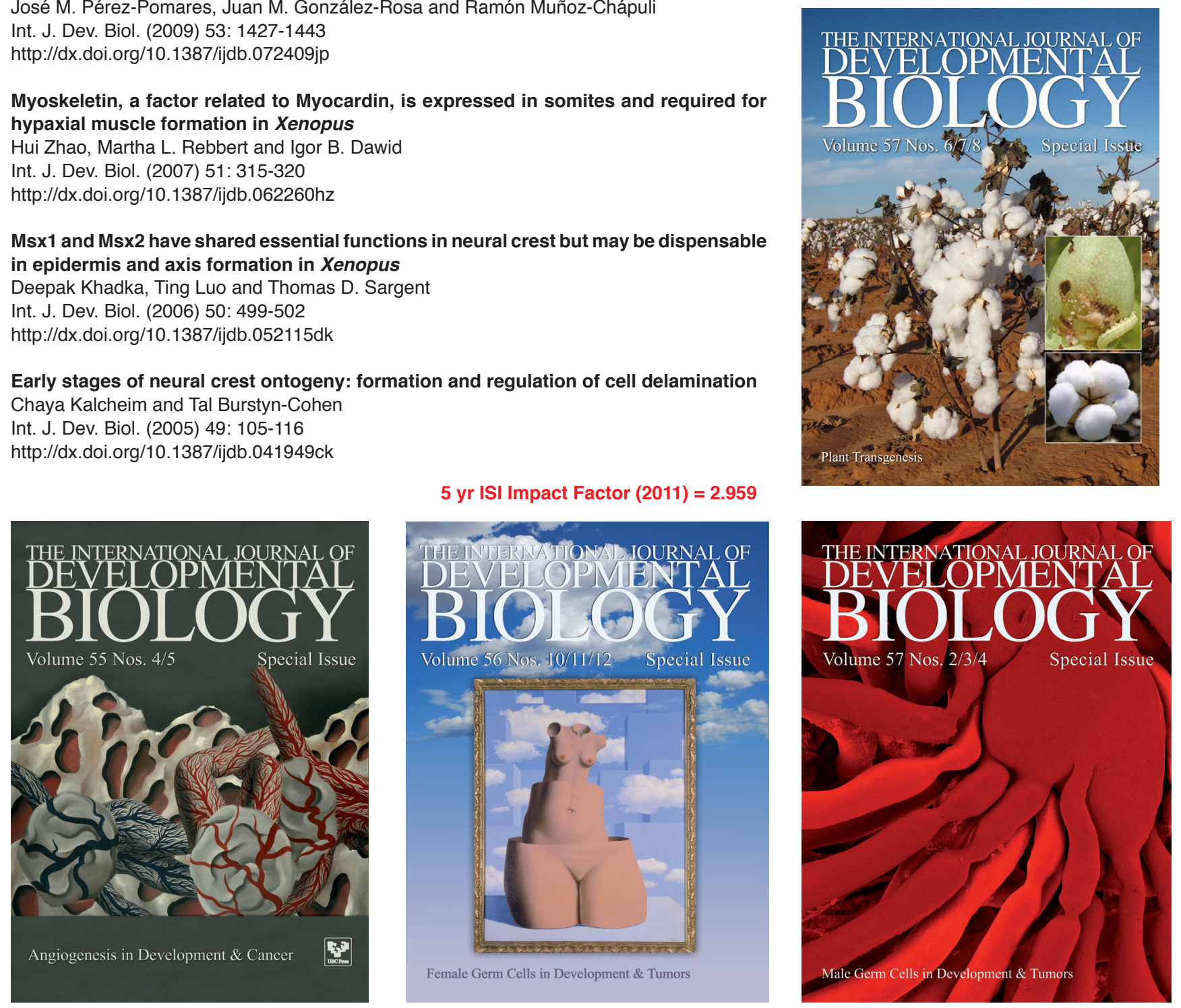